\title{
Photodetectors Based on Micro-nano Structure Material
}

\author{
Yu Yu ${ }^{1,2 *}$, Wuyue Wang ${ }^{1,2}$, Weihua $L^{3}{ }^{3}$, Gong Wang ${ }^{1,2}$, Yulei Wang ${ }^{1,2}$, Zhiwei Lu ${ }^{1,2}$, Sensen $L^{4}{ }^{4}$, \\ Wanli Zhao ${ }^{4}$, Yuhai $\mathrm{Li}^{4}$, Tongyu $\mathrm{Liu}^{4}$ and Xiusheng Yan ${ }^{4}$ \\ ${ }^{1}$ Center for Advanced Laser Technology, Hebei University of Technology, Tianjin, China, ${ }^{2}$ Hebei Key Laboratory of Advanced \\ Laser Technology and Equipment, Tianjin, China, ${ }^{3}$ Weihai Photonics Information Technology Lab Co., Ltd., Shandong, China, \\ ${ }^{4}$ Science and Technology on Electro-Optical Information Security Control Laboratory, Tianjin, China
}

Photodetectors converting optical signals into electrical signals have been widely utilized and have received more and more attention in scientific research and industrial fields including optical interconnection, optical communication, and environmental monitoring. Herein, we summarize the latest development of photodetectors with different micro-nano structures and different materials and the performance indicators of photodetectors. Several photodetectors, such as flexible, ultraviolet two-dimensional (2D) microscale, and dual-band photodetectors, are listed in this minireview. Meanwhile, the current bottleneck and future development prospects of the photodetector are discussed.

OPEN ACCESS

Edited by:

Yue-Feng Liu,

Jilin University, China

Reviewed by:

Jingjing Zhang,

National University of Defense

Technology, China

Xiaoxian Song,

Jiangsu University, China

*Correspondence:

Yu Yu

yuyu1990@hebut.edu.cn

Specialty section:

This article was submitted to Nanoscience,

a section of the journal

Frontiers in Chemistry

Received: 09 December 2021 Accepted: 24 December 2021

Published: 12 January 2022

Citation: YuY, Wang W, LiW, Wang G, Wang Y, LU Z, LiS, Zhao W, LiY, Liu T and YanX (2022) Photodetectors Based on Micro-nano Structure Material.

Front. Chem. 9:832028

doi: 10.3389/fchem.2021.832028
Keywords: photodetector, micro-nano structure, performance, fabrication, applications

\section{INTRODUCTION}

Traditionally, many ordered micro-nano structures have been emerged in natural organisms. Particularly, for plants, the micro-nano structures can guide water droplets to roll freely on their leaves or stay on petals (Zhang et al., 2012a; Zhang et al., 2012b). Inspired by the micro-nano structure of organisms in nature, a growing number of researchers have focused on the application of micro-nano structures in scientific research. Micro-nano structure arrays are widely used in PDs due to their unique order-related characteristics of ordering or patterning. Photodetectors (PDs) that convert optical signals into electrical signals have been widely used and paid more and more attention in scientific research and industrial fields such as biological detection, optical communications, and environmental monitoring (Teng et al., 2018). At present, the main materials used in photodetectors for detecting ultraviolet (UV) to near-infrared spectra are crystalline-Si and III-V (Deng et al., 2015). The reason why micro-nano structure arrays can be rapidly developed in the fields of science and industry is because they can improve the efficiency of light scattering, reduce light reflection efficiency, and extract light better and surface-tovolume ratio of organic light-emitting devices (OLED) (Gao et al., 2021), resulting in the photon supersurface (Li et al., 2021). Different performances can be achieved by changing the size, distribution and different result shapes of each micro/nano structure.

At present, the production of photodetectors with different properties mainly depends on the development of nanotechnology, including template method, photolithography, self-assembly and other methods. Many new types of photodetectors have been fabricated through the above technology, such as high-sensitivity phototransistors (Hai et al., 2015), flexible photodetectors, self-powered ultraviolet detectors with heterojunction nanowire arrays, pyramid array photodetectors, and dual-band detection array van der Waals. Broadband detector, sensitive infrared photodetector (IRPD). Flexible photodetectors (PD) have received more and more attention due to their structural characteristics in many applications, such as wearable 


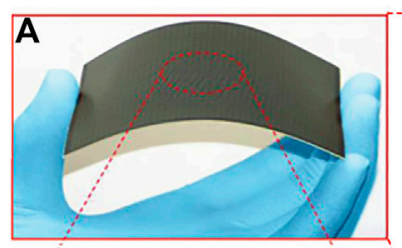

B

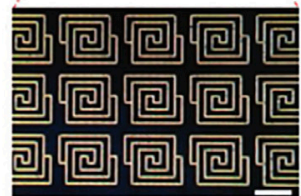

D

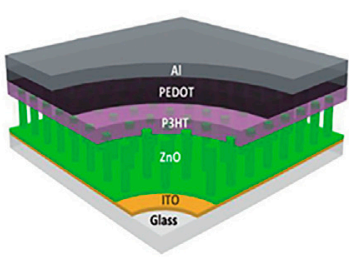

C

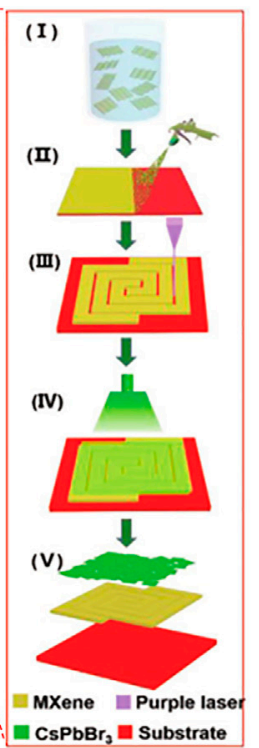

E

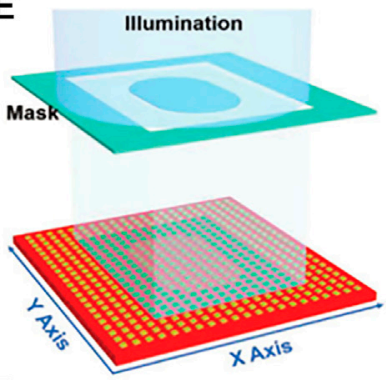

$\mathbf{F}$

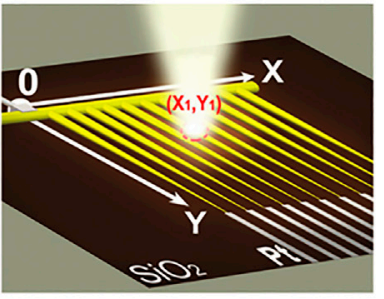

FIGURE 1 | (A) Exhibition of large-area arrays MXene electrode. (B) Micro pattern corresponding to (A). (C) Schematic diagram of flexible photodetector processing process. (D) Schematic of the P3HT/ZnO heterojunction photodetector. (E) Schematic diagram of a digital sensor based on a large-area array photodetector. (F) Schematic diagram of the CdS branched photodetector.

optoelectronic devices, bendable imaging sensors and implanted optoelectronic devices (Deng et al., 2019). The self-powered photodetector array based on organic-inorganic heterojunction has unique flexibility and stability due to its special structure, which makes it widely used in optical imaging (Ouyang et al., 2017). Among them, sapphire is used as the substrate, and the pyramidal structure of $\mathrm{MoS}_{2}$ as the material can enhance the strong interaction, thereby improving the performance of the optoelectronic device (Wang P et al., 2017). Dual-band photodetector for dual-color imaging. By introducing a strong local field, the dark current is reduced, so that the photogenerated carrier separation efficiency is increased, so that the dual-band detection capability of the broadband photodetector is improved (Wang $S$ et al., 2017). Each structure has its own unique properties, and photodetectors of different structure types are used in different optical fields. Therefore, it is particularly important to study the characteristics of different structures in photodetectors.

In this minireview, we focus on the latest developments in the application of different structures to photodetectors. Summarized typical cases, such as flexible photodetectors, ultraviolet photodetectors, high-sensitivity ultra-fast response array photodetectors, high-performance dual-band photodetectors, are discussed the challenges faced by photodetectors and future prospects.

\section{FLEXIBLE PHOTODETECTORS BASED ON MICRO-NANO STRUCTURE MATERIAL}

Flexible photodetectors (FPDs) have received more and more attention due to their structural characteristics in many applications, such as Optical communication, industrial automatic control, medical sensor monitoring and intelligent robots and implanted optoelectronic devices. For large-scale manufacturing of FPD processing technology and its use of complex high-vacuum technology, which makes the functional function of its active materials need to achieve a good match. Traditional PDs are usually fabricated on rigid substrates, which seriously hinders their rapid development in the fields of flexible optoelectronic devices such as industrial automatic control, medical sensor monitoring, and implantable optoelectronic devices (Liu et al., 2017). In contrast to rigid PDs, FPDs can meet the needs of industrial automatic control, medical sensor monitoring, and implantable optoelectronic devices. However, it has high optoelectronic performance and good flexibility. It is integrated in an optoelectronic device, which is still a huge challenge for technical processing so far (Hossain et al., 2018). In 2019, Deng et al. demonstrated all spray processable and large area FPDs on plain paper based on two-dimensional (2D) $\mathrm{CsPbBr}_{3}$ nanosheets and conductive $\mathrm{Ti}_{3} \mathrm{C}_{2} \mathrm{~T}_{\mathrm{x}}$ (MXene) (Deng et al., 2019). Figure 1A is its large-area MXene electrode array, and Figure $\mathbf{1 B}$ is its microscopic model. The production process is shown in Figure 1C. Due to the Figure 1C good conductivity of MXene, the higher crystallinity of $2 \mathrm{D} \mathrm{CsPbBr}$, and their good matching with the work function, on/off current ratio of the PD reaches $2.3 \times 10^{3}$, and the light response reaches $18 \mathrm{~ms}$. At the same time, Jones' detection rate $\left(\mathrm{D}^{*}\right)$ reached $6.4 \times 10^{8}$ under $10 \mathrm{~V}$ bias and the response rate $(\mathrm{R})$ reached $44.9 \mathrm{~mA} \mathrm{~W}^{-1}$. In addition, the PD still has good flexibility and stability after being bent 1,500 times. 


\section{UV MICRO-NANO STRUCTURE MATERIAL PHOTODETECTORS}

As an electronic sensor that converts electrical signals into light signals, ultraviolet (UV) photodetectors have good application prospects in many ways such as medicine, biology, flame monitoring, optical communication, day/night monitoring, and missile detection (Koppens et al., 2014; Chen et al., 2016; Liang et al., 2016; Esopi et al., 2017; Zhang et al., 2017). In 2017, Bangsen et al. have constructed a self-powered UV photodetector based on p-P3HT/n-ZnO nanowire array heterojunction (Ouyang et al., 2017). The schematic diagram of the photodetector is shown in Figure 1D. The light response of this PDs at $\lambda=365 \mathrm{~nm}$ can reach $125 \mu \mathrm{A} \mathrm{W}^{-1}$ to $0.84 \mathrm{~mW} \mathrm{~cm}^{-2}$, and its response and recovery time are both less than $100 \mathrm{~ms}$. Moreover, a photodetector array with 16 pixels is successfully demonstrated for light imaging of complex patterns, such as number-shape and cross-shape. This study provides a practical solution to achieve large-scale UV imaging by integrating inorganic-organic hybrid photodetector into selfpowred array configuration.

\section{D MICROSCALE POSITION-SENSITIVE PHOTODETECTORS}

With the continuous improvement of integration and the reduction of the size of nano-devices, two-dimensional micro-scale position sensitive detectors (PSD) have received more and more attention. Nowadays, two-dimensional materials have gradually become mainstream due to their excellent optoelectronic properties (An et al., 2013; Gan et al., 2013; Lopez-Sanchez et al., 2013; Wang et al., 2013; Baugher et al., 2014; Furchi et al., 2014a; Furchi et al., 2014b; Gong et al., 2014; Lee et al., 2014; Ross et al., 2014; Zhang et al., 2014; Li et al., 2015; Wang et al., 2015a; Wang et al., 2015b; Youngblood et al., 2015; Yue et al., 2015; Long et al., 2016). Figure 1E is a schematic diagram of a digital sensor based on a large area array PD. In 2018. For the first time, Chen et al. have grown a well-defined hollow spherical nanoshell array of two-dimensional transition metal aluminum dichloride (TMDC) nanomaterials for $\mathrm{MoSe}_{2}$ and $\mathrm{MoS}_{2}$ through chemical vapor deposition technology (Chen et al., 2018). The responsivity of the $\mathrm{MoSe}_{2}$ hollow sphere photodetector reaches 8.9 $\mathrm{A} \mathrm{W}^{-1}$, which is about 10 times that of the $\mathrm{MoSe}_{2}$ dense film $\left(0.9 \mathrm{~A} \mathrm{~W}^{-1}\right)$. At the same time, the hollow sphere PDs has a fast response and recovery speed and $\lambda=365 \mathrm{~nm}$ irradiation good durability at wavelengths. $\mathrm{Xu}$ et al. designed a high-sensitivity ultra-fast response array photodetector based on a new twodimensional lead iodide perovskite crystal ( $\mathrm{Xu}$ et al., 2019). The array photodetector achieves high phodetectivities $\left(6.3 \times 10^{12}\right.$ Jones $)$ responsivities $\left(\approx 47 \mathrm{~A} \mathrm{~W}^{-1}\right)$ and low dark current $\left(\approx 2.4 \times 10^{-11} \mathrm{~A}\right)$. In 2019, Li et al. developed a method capable of heterogeneous integration of atomically thin 2D crystal arrays for system-onchip electrons on a planar patterned silicon substrate ( $\mathrm{Li}$ et al., 2019). In addition, multi-channel devices with good optical and electrical characteristics are widely used in system-on-chip (Hao et al., 2019). Schematic diagram of CdS branch photodetector. As shown in Figure 1F. Hao et al. based on a highly ordered combshaped CdS nanowire array with tapered branches, a one-step synthesis strategy is used to achieve high-resolution $2 \mathrm{D}$ positionsensitive photodetection through the variable resistance of multiple lines and the variable optical response of different parts Device. The tapered branch can accurately identify the position of the incident light in each area of the nanowire array according to the change of the photocurrent. In addition to the above-mentioned traditional photodetectors, more and more complex photodetectors are gradually being used in many fields. Multicolor photodetectors have a wide range of applications in the fields of imaging, (Schermelleh et al., 2008; Sang et al., 2013), medical treatment, (Keller et al., 2001), astronomical observations (Fontana et al., 2004) and military applications (Tribolet and Destefanis, 2005). Ji et al. used interface engineering technology to develop an ultravioletvisible multicolor photodetector based on $\mathrm{n}-\mathrm{Si}(111) / \mathrm{TiO}_{2}$ nanorod array heterojunction (Tao et al., 2016). The photodetector is manufactured through continuous processes such as chemical etching, magnetron sputtering, hydrothermal growth and assembly. In the case of low reverse bias voltage (0 $2 \mathrm{~V}$ ), only photo-generated electrons in $\mathrm{TiO}_{2}$ can pass through the low $\triangle \mathrm{EC}$ barrier, and the device only responds to ultraviolet light.

\section{DUAL-BAND MICRO-NANO STRUCTURE MATERIAL PHOTODETECTORS}

In the past few decades, in the field of dual-color detection technology (DCDT), people have made significant progress by introducing new materials such as quantum dots and superlattices (Martyniuk et al., 2014; Kufer et al., 2015; Lei et al., 2015; Hoang et al., 2016). Wang et al. designed a dualband photodetector with high-performance dual-color imaging and wafer-level 2D GaSe/GaSb van der Waals vertical heterostructure based on molecular beam epitaxial growth (Wang P et al., 2017). By introducing a strong local field, the dark current is reduced, so that the photo-generated carrier separation efficiency is increased, so that the dual-band detection capability of the broadband photodetector is improved. Ultrasensitive visible and infrared specific detectivities reach up to $2.2 \times 10^{12}$ and $1.3 \times 10^{12}$ Jones, respectively, and an excellent external quantum efficiency up to $50 \%$ is obtained with microsecond response speed, which is expected due to its photovoltaic mechanism for free-carrier generation. This new type of heterogeneous photodiode also has the good photoresponsibility of the two-dimensional material GaSe in the visible light band and also have the excellent photodetection performance of the traditional GaSb in the infrared light band. It provides a new way for two-dimensional materials to be used in actual room temperature applications.

\section{CONCLUSION AND OUTLOOK}

In this minireview, we summarize the performance indicators of photodetectors with different structures. Such as flexible photodetectors, ultraviolet photodetectors, high-sensitivity ultra-fast response array photodetectors, and high-performance dual-band photodetectors are listed. Although these devices have 
reached very good performance indicators. However, there are still many challenges to realize low-cost and large-scale preparation. However, with the rapid development of micronano manufacturing technology, photodetectors with good performance will be better used in electronic information, optical communications, environmental monitoring and other fields.

\section{AUTHOR CONTRIBUTIONS}

YY, GW, and WL contributed to conception and design of the study. YY, WW, YW, and ZL organized the database. SL, WZ, YL,

\section{REFERENCES}

An, X., Liu, F., Jung, Y. J., and Kar, S. (2013). Tunable Graphene-Silicon Heterojunctions for Ultrasensitive Photodetection. Nano Lett. 13, 909-916. doi:10.1021/nl303682j

Baugher, B. W. H., Churchill, H. O. H., Yang, Y., and Jarillo-Herrero, P. (2014). Optoelectronic Devices Based on Electrically Tunable P-N Diodes in a Monolayer Dichalcogenide. Nat. Nanotech. 9, 262-267. doi:10.1038/ nnano.2014.25

Chen, H., Liu, H., Zhang, Z., Hu, K., and Fang, X. (2016). Nanostructured Photodetectors: From Ultraviolet to Terahertz. Adv. Mater. 28, 403-433. doi:10.1002/adma.201503534

Chen, X., Yang, H., Liu, G., Gao, F., Dai, M., Hu, Y., et al. (2018). Hollow Spherical Nanoshell Arrays of 2D Layered Semiconductor for High-Performance Photodetector Device. Adv. Funct. Mater. 28 (8), 1705153-117051539. doi:10.1002/adfm.201705153

Deng, H., Yang, X., Dong, D., Li, B., Yang, D., Yuan, S., et al. (2015). Flexible and Semitransparent Organolead Triiodide Perovskite Network Photodetector Arrays With High Stability. Nano Lett. 15, 7963-7969. doi:10.1021/ acs.nanolett.5b03061

Deng, W., Huang, H, Jin, H., Li, W., Chu, X., Xiong, D., et al. (2019). All-SprayedProcessable, Large-Area, and Flexible Perovskite/MXene-Based Photodetector Arrays for Photocommunication. Adv. Opt. Mater. 7 (6), 1801521. doi:10.1002/ adom.201801521

Esopi, M. R., Calcagno, M., and Yu, Q. (2017). Organic Ultraviolet Photodetectors Exhibiting Photomultiplication, Low Dark Current, and High Stability. Adv. Mater. Technol. 2, 1700025. doi:10.1002/admt.201700025

Fontana, A., Pozzetti, L., Donnarumma, I., Renzini, A., Cimatti, A., Zamorani, G., et al. (2004). The K20 Survey. AઐA. 424, 23-42. doi:10.1051/0004-6361: 20035626

Furchi, M. M., Polyushkin, D. K., Pospischil, A., and Mueller, T. (2014a). Mechanisms of Photoconductivity in Atomically Thin MoS2. Nano Lett. 14, 6165-6170. doi:10.1021/nl502339q

Furchi, M. M., Pospischil, A., Libisch, F., Burgdörfer, J., and Mueller, T. (2014b). Photovoltaic Effect in an Electrically Tunable van der Waals Heterojunction. Nano Lett. 14, 4785-4791. doi:10.1021/nl501962c

Gan, X., Shiue, R.-J., Gao, Y., Meric, I., Heinz, T. F., Shepard, K., et al. (2013). ChipIntegrated Ultrafast Graphene Photodetector with High Responsivity. Nat. Photon. 7, 883-887. doi:10.1038/nphoton.2013.253

Gao, X.-m., Liu, Y. F., Liu, Y.-f., Zhang, H.-j., Zhang, T.-r., Bi, Y.-g., et al. (2021). Nanoimprinted Structures for Organic Light-Emitting Devices and Lasers. Chin. J. Liq. Cryst. Disp. 36 (1), 8-20. doi:10.37188/cjlcd.2020-0277

Gong, Y., Lin, J., Wang, X., Shi, G., Lei, S., Lin, Z., et al. (2014). Vertical and InPlane Heterostructures from WS2/MoS2 Monolayers. Nat. Mater. 13, 1135-1142. doi:10.1038/nmat4091

Hai, H., Peng, W., Gao, Y., Wang, X., Lin, T., Wang, J., et al. (2015). Highly Sensitive Phototransistor Based on Gase Nanosheets. Appl. Phys. Lett. 44 (14), 699. doi:10.1063/1.4933034

Hao, Y., Guo, S., Weller, D., Zhang, M., Ding, C., Chai, K., et al. (2019). Positionsensitive Array Photodetector Based on Comb-Like Cds Nanostructure With Cone-
TL, and XY performed the statistical analysis. YY and WW wrote the first draft of the manuscript. YY, GW and WW wrote sections of the manuscript. All authors contributed to manuscript revision, read, and approved the submitted version.

\section{FUNDING}

This work was supported by the National Natural Science Foundation of China (Grant Nos. 62005074, 61927815, 62075056, and 62004059), Natural Science Foundation of Hebei Province (No. F2021202002) and Key Laboratory Fund Project (No. 61421070302).

Shape Branches. Adv. Funct. Mater. 29 (1), 1805967-11805967. doi:10.1002/ adfm.201805967

Hoang, A. M., Dehzangi, A., Adhikary, S., and Razeghi, M. (2016). High Performance Bias-Selectable Three-Color Short-Wave/Mid-Wave/LongWave Infrared Photodetectors Based on Type-II InAs/GaSb/AlSb Superlattices. Sci. Rep. 6, 24144. doi:10.1038/srep24144

Hossain, M., Kumar, G. S., Barimar Prabhava, S. N., Sheerin, E. D., McCloskey, D., Acharya, S., et al. (2018). Transparent, Flexible Silicon Nanostructured Wire Networks with Seamless Junctions for High-Performance Photodetector Applications. ACS Nano. 12, 4727-4735. doi:10.1021/ acsnano.8b01387

Keller, P., Toomre, D., Díaz, E., White, J., and Simons, K. (2001). Multicolour Imaging of Post-Golgi Sorting and Trafficking in Live Cells. Nat. Cell Biol. 3, 140-149. doi:10.1038/35055042

Koppens, F. H. L., Mueller, T., AvourisFerrari, P. A. C., Ferrari, A. C., Vitiello, M. S., and Polini, M. (2014). Photodetectors Based on Graphene, Other Two-Dimensional Materials and Hybrid Systems. Nat. Nanotech. 9, 780-793. doi:10.1038/nnano.2014.215

Kufer, D., Nikitskiy, I., Lasanta, T., Navickaite, G., Koppens, F. H. L., and Konstantatos, G. (2015). Hybrid 2D-0D MoS2-PbS Quantum Dot Photodetectors. Adv. Mater. 27, 176-180. doi:10.1002/adma.201402471

Lee, C.-H., Lee, G.-H., van der Zande, A. M., Chen, W., Li, Y., Han, M., et al. (2014). Atomically Thin p-n Junctions With van der Waals heterointerfaces. Nat. Nanotech. 9, 676-681. doi:10.1038/nnano.2014.150

Lei, W., Antoszewski, J., and Faraone, L. (2015). Progress, Challenges, and Opportunities for HgCdTe Infrared Materials and Detectors. Appl. Phys. Rev. 2, 41303. doi:10.1063/1.4936577

Li, H.-M., Lee, D., Qu, D., Liu, X., Ryu, J., Seabaugh, A., et al. (2015). Ultimate Thin Vertical P-N Junction Composed of Two-Dimensional Layered Molybdenum Disulfide. Nat. Commun. 6, 6564. doi:10.1038/ ncomms 7564

Li, H., Gu, S., Zhang, Q., Song, E., Kuang, T., Chen, F., et al. (2021). Recent Advances in Biofluid Detection with Micro/Nanostructured Bioelectronic Devices. Nanoscale. 13 (6), 3436-3453. doi:10.1039/d0nr07478k

Li, S. L., Zhang, L., Zhong, X., Gobbi, M., Bertolazzi, S., Guo, W., et al. (2019). Nano-subsidence Assisted Precise Integration of Patterned TwoDimensional Materials for High-Performance Photodetector Arrays. Acs Nano. 13 (2), 2654-2662. doi:10.1021/acsnano.9b00889

Liang, Y., Wang, Y., Wang, J., Wu, S., Jiang, D., and Lian, J. (2016). HighPerformance Flexible Photodetectors Based on Single-Crystalline Sb2Se3 Nanowires. RSC Adv. 6, 11501-11506. doi:10.1039/c5ra23542a

Liu, X., Yu, D., Cao, F., Li, X. J., Ji, J., Chen, J., et al. (2017). Low-Voltage Photodetectors with High Responsivity Based on Solution-Processed Micrometer-Scale All-Inorganic Perovskite Nanoplatelets. Small. 13, 1700364. doi:10.1002/smll.201700364

Long, M., Liu, E., Wang, P., Gao, A., Xia, H., Luo, W., et al. (2016). Broadband Photovoltaic Detectors Based on an Atomically Thin Heterostructure. Nano Lett. 16, 2254-2259. doi:10.1021/acs.nanolett.5b04538

Lopez-Sanchez, O., Lembke, D., Kayci, M., Radenovic, A., and Kis, A. (2013). Ultrasensitive Photodetectors Based on Monolayer MoS2. Nat. Nanotech. 8, 497-501. doi:10.1038/nnano.2013.100 
Martyniuk, P., Antoszewski, J., Martyniuk, M., Faraone, L., and Rogalski, A. (2014). New Concepts in Infrared Photodetector Designs. Appl. Phys. Rev. 1, 41102. doi:10.1063/1.4896193

Ouyang, B., Zhang, K., and Yang, Y. (2017). Self-Powered UV Photodetector Array Based on P3HT/ZnO Nanowire Array Heterojunction. Adv. Mater. Technol. 2, 1700208. doi:10.1002/admt.201700208

Ross, J. S., Klement, P., Jones, A. M., Ghimire, N. J., Yan, J., Mandrus, D. G., et al. (2014). Electrically Tunable Excitonic Light-Emitting Diodes Based on Monolayer WSe2 P-N Junctions. Nat. Nanotech. 9, 268-272. doi:10.1038/nnano.2014.26

Sang, L., Hu, J., Zou, R., Koide, Y., and Liao, M. (2013). Arbitrary Multicolor Photodetection by Hetero-Integrated Semiconductor Nanostructures. Sci. Rep. 3, 2368. doi: $10.1038 /$ srep02368

Schermelleh, L., Carlton, P. M., Haase, S., Shao, L., Winoto, L., Kner, P., et al. (2008). Subdiffraction Multicolor Imaging of the Nuclear Periphery with 3D Structured Illumination Microscopy. Science. 320, 1332-1336. doi:10.1126/ science.1156947

Tao, J., Qian, L., Rujia, Z., Yangang, S., Kaibing, X., Liwen, S., et al. (2016). An Interface Engineered Multicolor Photodetector Based on $\mathrm{N}-\mathrm{si}(111) / \mathrm{Tio}_{2}$ Nanorod Array Heterojunction. Adv. Funct. Mater. 26 (9), 1400-1410. doi:10.1002/adfm.201504464

Teng, F., Hu, K., Ouyang, W., and Fang, X. (2018). Photoelectric Detectors Based on Inorganic p-Type Semiconductor Materials. Adv. Mater. 30, 1706262. doi:10.1002/adma.201706262

Tribolet, P., and Destefanis, G. (2005). Third Generation and Multicolor IRFPA Developments: a Unique Approach Based on DEFIR (Invited Paper). Conf. Infrared Technol. Appl. 5783, 350. doi:10.1117/12.607544

Wang, K., Wang, J., Fan, J., Lotya, M., O’Neill, A., Fox, D., et al. (2013). Ultrafast Saturable Absorption of Two-Dimensional MoS2 Nanosheets. ACS Nano. 7, 9260-9267. doi:10.1021/nn403886t

Wang, P., Liu, S., Luo, W., Fang, H., Gong, F., Guo, N., et al. (2017). Arrayed Van Der Waals Broadband Detectors for Dual-Band Detection. Adv. Mater. 29 (16), 1604439. doi:10.1002/adma.201604439

Wang, S., Medina, H., Hong, K.-B., Wu, C.-C., Qu, Y., Manikandan, A., et al. (2017). Thermally Strained Band Gap Engineering of Transition-Metal Dichalcogenide Bilayers with Enhanced Light-Matter Interaction toward Excellent Photodetectors. ACS Nano 11 (9), 8768-8776. doi:10.1021/ acsnano.7b02444

Wang, X., Jiang, X., Wang, T., Shi, J., Liu, M., Zeng, Q., et al. (2015a). Electrically Configurable Graphene Field-Effect Transistors with a Graded-Potential Gate. Nano Lett. 15, 3212-3216. doi:10.1021/ acs.nanolett.5b00396

Wang, X., Wang, P., Wang, J., Hu, W., Zhou, X., Guo, N., et al. (2015b). Ultrasensitive and Broadband MoS2Photodetector Driven by Ferroelectrics. Adv. Mater. 27, 6575-6581. doi:10.1002/adma.201503340
Xu, Z., Li, Y., Liu, X., Ji, C., Chen, H., Li, L., et al. (2019). Highly Sensitive and Ultrafast Responding Array Photodetector Based on a Newly Tailored 2d lead Iodide Perovskite crystal. Adv. Opt. Mater. 7 (11), 1900308-11900308. doi:10.1002/adom.201900308

Youngblood, N., Chen, C., Koester, S. J., and Li, M. (2015). Waveguide-Integrated Black Phosphorus Photodetector with High Responsivity and Low Dark Current. Nat. Photon. 9, 247-252. doi:10.1038/nphoton.2015.23

Yue, R., Barton, A. T., Azcatl, A., Pena, L. F., Wang, J., Peng, X., et al. (2015). HfSe2 Thin Films: 2D Transition Metal Dichalcogenides Grown by Molecular Beam Epitaxy. ACS Nano. 9, 474-480. doi:10.1021/nn5056496

Zhang, K., Peng, M., Wu, W., Guo, J., Gao, G., Liu, Y., et al. (2017). A Flexible $\mathrm{P}-\mathrm{CuO} / \mathrm{n}-\mathrm{MoS} 2$ Heterojunction Photodetector With Enhanced Photoresponse by the Piezo-Phototronic Effect. Mater. Horiz. 4, 274-280. doi:10.1039/ c6mh00568c

Zhang, W., Chiu, M.-H., Chen, C.-H., Chen, W., Li, L.-J., and Wee, A. T. S. (2014). Role of Metal Contacts in High-Performance Phototransistors Based on WSe2 Monolayers. ACS Nano. 8, 8653-8661. doi:10.1021/nn503521c

Zhang, Y.-L., Chen, Q.-D., Jin, Z., Kim, E., and Sun, H.-B. (2012a). Biomimetic Graphene Films and Their Properties. Nanoscale. 4 (16), 4858-4869. doi:10.1039/C2NR30813D

Zhang, Y.-L., Xia, H., Kim, E., and Sun, H.-B. (2012b). Recent Developments in Superhydrophobic Surfaces with Unique Structural and Functional Properties. Soft Matter. 8 (44), 11217-11231. doi:10.1039/C2SM26517F

Conflict of Interest: Author WL is employed by Weihai Photonics Information Technology Lab Co., Ltd.

The remaining authors declare that the research was conducted in the absence of any commercial or financial relationships that could be construed as a potential conflict of interest.

Publisher's Note: All claims expressed in this article are solely those of the authors and do not necessarily represent those of their affiliated organizations, or those of the publisher, the editors and the reviewers. Any product that may be evaluated in this article, or claim that may be made by its manufacturer, is not guaranteed or endorsed by the publisher.

Copyright (C) $2022 \mathrm{Yu}$, Wang, Li, Wang, Wang, Lu, Li, Zhao, Li, Liu and Yan. This is an open-access article distributed under the terms of the Creative Commons Attribution License (CC BY). The use, distribution or reproduction in other forums is permitted, provided the original author(s) and the copyright owner(s) are credited and that the original publication in this journal is cited, in accordance with accepted academic practice. No use, distribution or reproduction is permitted which does not comply with these terms. 